\title{
Distortion Reduction in Fractional Delay Filters
}

\author{
Zijian Zhou, Yuli Yang, Senior Member, IEEE, Meng Ma, and Bingli Jiao, Senior Member, IEEE
}

\begin{abstract}
As the digital version of a continuous-time delay, the concept of fractional delay (FD) is exploited to approximate a desired delay that is not a multiple of the sampling interval. However, in FD filters, there is always a severe distortion at the beginning of delayed signals, referred to as head distortion. This letter identifies the cause of head distortion and proposes a solution to this problem for reducing the overall distortion in FD filters. For the purpose of performance evaluation, relative rootmean-square (RMS) error is formulated as a metric to quantify the overall difference between the frequency-domain response of an FD filter and the ideal one. Moreover, illustrative numerical results on the proposed scheme applied in FD filters with classical sinc, Farrow and Lagrange interpolation substantiate the validity and feasibility of our solution.
\end{abstract}

Index Terms-Fractional delay (FD) filters, head distortion, sinc interpolation, Farrow structure, Lagrange interpolation, root-mean-square (RMS).

\section{INTRODUCTION}

To generate the desired delays that are not a multiple of the sampling interval, fractional delay (FD) techniques have been exploited and playing an essential role in digital signal processing for a wide range of applications over the past decades [1]-[3]. Since the readily feasible delay in uniformly sampled digital systems is always a multiple of the shortest possible time, i.e., equal to the sampling interval, the FD concept is used to remedy the gap between the desired delay that includes a fraction of the sampling interval and its closest multiple of the sampling interval, through interpolation. Several interpolation approaches, e.g., sinc [1], [4], Lagrange [5], [6], B-spline [7], [8], Farrow structure [9], and Newton structure [10], [11], are adopted to form FD filters.

However, there is a severe distortion at the beginning of the FD filter output, referred to as head distortion, mainly owing to the null input for this part, which leaves a dilemma in the design of FD filters. To increase the FD filter's order reduces the height of ripples and sharpens the drop-off in the Gibbs phenomenon [12]. On the other hand, the higher order of FD filter results in more null input at the beginning of its output and, hence, leading to worse distortion.

Specifically, FD filters of high order are designed to guarantee the construction accuracy of delayed signals in wireless communication systems [13]. For a co-frequency co-time full-duplex (CCFD) transceiver, FD filters are exploited to reconstruct a delayed version of transmitted signals at the receiver port for cancelling the self-interference (SI) [14], [15], where the entire waveform of a delayed signal is expected to be exactly the same as that of the transmitted signal [16].

Z. Zhou, M. Ma and B. Jiao are with the Department of Electronics, Peking University, Beijing 100871, China (e-mail: zjzhou1008@pku.edu.cn, mam@pku.edu.cn, jiaobl@pku.edu.cn).

Y. Yang is with the School of Engineering, University of Lincoln, Lincoln LN6 7TS, U.K. (e-mail: yyang@lincoln.ac.uk).
However, the head part of a delayed signal has to suffer from the distortion caused by a large section of null input in the reconstruction, even if the SI channel state information is perfectly known at the transceiver. The head distortion leads to high residue SI and badly degrades the CCFD communications, especially for the delivery of short-burst signals [17], [18]. Motivated by this, we propose a solution to the head distortion problem for improving the performance of FD filters. To the best of our knowledge, this problem has not yet been considered before. In particular, our main contributions in this work are threefold:

- The head distortion problem in FD filters is studied and its cause is analyzed, using sinc and Lagrange interpolation FD filters as two examples.

- A solution to the head distortion problem is proposed for reducing the overall difference between the FD filter output and the desired version.

- Relative root-mean-square (RMS) error is formulated to quantify the overall difference between the frequencydomain response of an FD filter and the ideal one, which verifies the validity and feasibility of our solution.

The remainder of this letter is organized as follows. Section II presents the head distortion problem and Section III proposes the solution to this problem. The performance of the proposed scheme is evaluated in Section IV, and this work is concluded in Section V.

The following mathematical notations are used: Boldface uppercase and lowercase letters denote matrices and vectors, respectively. The transpose operator is denoted by $(\cdot)^{\mathrm{T}}$, and the root-mean-square (RMS) calculator is denoted by $\|\cdot\|$. The greatest and least integer functions are represented by $\lfloor\cdot\rfloor$ and $\lceil\cdot\rceil$, respectively.

\section{HeAd Distortion}

\section{A. System Model}

Consider a continuous-time signal $x_{c}(t)$ is bandlimited in the range of $\left[0, f_{0}\right]$ and expressed by its $L+1$ samples $x\left(l T_{s}\right)$, where $l=0,1, \cdots, L$ is the sample index and $T_{s}$ is the sampling interval, i.e., the inverse of the sampling rate $f_{s} \geqslant 2 f_{0}$. For convenience of notation, we omit $T_{s}$ and use $x(l)$ to denote the samples of the discrete-time signal.

The discrete-time version of the FD operation for a sampled bandlimited signal can be written as

$$
\tilde{y}(l)=x(l-D),
$$

where $\tilde{y}(l)$ is the desired discrete-time output, $l=0,1, \cdots, L$. The desired delay $D=\lfloor D\rfloor+\alpha$, where $\lfloor D\rfloor$ is the integer part and $\alpha \in[0,1]$ is the FD.

The sample values of $\tilde{y}(l)$ are then obtained via interpolation from the sequence $x(l)$. This interpolation is accomplished 


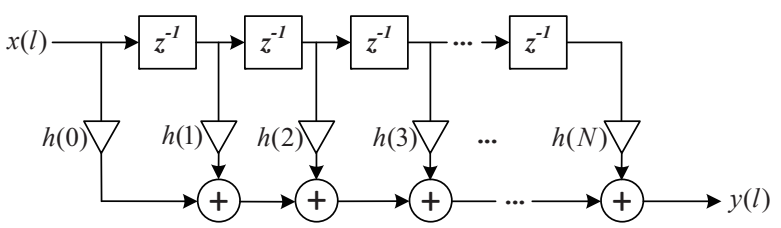

Fig. 1. A direct form discrete-time FIR filter of order $N$.

by utilizing a direct-form finite impulse response (FIR) FD filter of order $N<L$, as shown in Fig. 1, where $h(n), n=$ $0,1, \cdots, N$, are the real coefficients that shape the impulse response of the FD filter. The output samples of the FD filter are expressed by

$$
y(l)=\sum_{n=0}^{N} x(l-n) h(n), \quad l=0,1, \cdots, L .
$$

Although (2) holds for $L=\infty$, the sequence $x(l)$ is of finite length in practical applications. Therefore, (2) is readily written in a vector form as

$$
\mathbf{y}=\mathbf{X h},
$$

where the $(L+1) \times 1$ vector $\mathbf{y}=[y(0), y(1), \cdots, y(L)]^{\mathrm{T}}$, and the $(N+1) \times 1$ vector $\mathbf{h}=[h(0), h(1), \cdots, h(N)]^{\mathrm{T}}$. As the samples $x(l)=0$ for $l<0$, the $(L+1) \times(N+1)$ matrix $\mathbf{X}$ is given by

$$
\left[\begin{array}{ccccc}
x(0) & 0 & \cdots & 0 & 0 \\
x(1) & x(0) & \cdots & 0 & 0 \\
\vdots & \vdots & \ddots & \vdots & \vdots \\
x(N-1) & x(N-2) & \cdots & x(0) & 0 \\
x(N) & x(N-1) & \cdots & x(1) & x(0) \\
\vdots & \vdots & \ddots & \vdots & \vdots \\
x(L) & x(L-1) & \cdots & x(L-N+1) & x(L-N)
\end{array}\right] .
$$

As a consequence of the null input for the output $y(\nu)$ when $\nu \in[0, N-1]$, the filter of order $N$ is truncated in (3) to a filter of order $\nu$, i.e., the filter's coefficients are truncated from $N+1$ taps to $\nu+1$ taps. In this case, the output is calculated using $y(\nu)=\mathbf{x}_{\mathrm{T} r, \nu} \mathbf{h}_{\mathrm{T} r, \nu}$, where the $1 \times(\nu+1)$ vector $\mathbf{x}_{\mathrm{T} r, \nu}=[x(\nu), x(\nu-1), \cdots, x(0)]$ contains the first $\nu+1$ samples in the input sequence, and the $(\nu+1) \times 1$ vector $\mathbf{h}_{\mathrm{T} r, \nu}=[h(0), h(1), \cdots, h(\nu)]^{\mathrm{T}}$ contains the coefficients within a truncated FD filter of order $\nu$.

Hence, there is a serious distortion for the first $N$ output samples $y(l), 0 \leqslant l \leqslant N-1$, which is referred to as head distortion. As a result, the FD filter's impulse response vector $\mathbf{h}$ in (3) varies from a truncated version $\mathbf{h}_{\mathrm{T} r, l}$ for $l \in[0, N-1]$ to a normal one without truncation for $l \in[N, L]$, in obtaining the output $y(l)$.

\section{B. Problem Statement}

So far, the FD filters without truncation have been well studied in the literature, but the truncated part that results in the head distortion has been disregarded. Herein, we will investigate the effect of the head distortion on the overall difference between an FD filter and the ideal one.

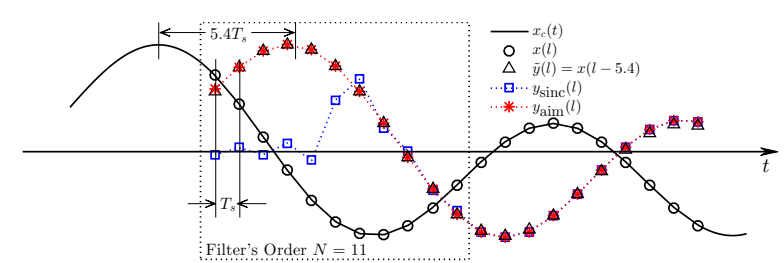

Fig. 2. Time-domain samples of the desired output $\tilde{y}(l)=x(l-5.4)$, the order $N=11$ sinc interpolation FD filter output $y_{\text {sinc }}(l)$, and our proposed solution's aimed output $y_{\text {aim }}(l)$.

Since the time-domain distortion caused by truncated FD filters can be mirrored from their frequency-domain responses, the frequency-domain error is used to measure the overall difference between the frequency-domain response of an FD filter and the ideal one, which is defined as

$$
\varepsilon(f)=\left|H(f)-e^{-j 2 \pi D f}\right|^{2},
$$

where $H(f)$ is the frequency-domain response of the considered FD filter, and $e^{-j 2 \pi D f}$ is the ideal frequency-domain response of an FD filter for the desired delay $D$.

In addition, the group delay is another metric to measure the time delay of an input signal's amplitude envelopes on various sinusoidal components through the FD filter, calculated using

$$
\tau(f)=-\frac{d \angle H(f)}{d f}
$$

Next, we use two classical interpolation paradigms to illustrate the head distortion problem in FD filters.

1) Sinc Interpolation: For the desired delay $D$, the FD filter coefficients with sinc interpolation are given in the form of sinc function as

$$
\begin{array}{r}
h_{\text {sinc }}(n, D)=\operatorname{sinc}(n-D)=\frac{\sin [\pi(n-D)]}{\pi(n-D)}, \\
n=0,1, \cdots, N .
\end{array}
$$

To illustrate the head distortion in time domain, we compare the desired delay signal $\tilde{y}(l)$ and the sinc interpolation FD filter output $y_{\text {sinc }}(l)$ in Fig. 2, where the desired delay $D=5.4$, i.e., $\tilde{y}(l)=x(l-5.4)$, and the FD filter is of order $N=11$. Our proposed solution aims at the output $y_{\text {aim }}(l)$, i.e., to reduce the head distortion suffered by FD filters for $l<11$.

2) Lagrange Interpolation: For the desired delay $D$, the FD filter coefficients with Lagrange interpolation are given in the form of Lagrange polynomial as

$$
h_{\text {Lag }}(n, D)=\prod_{k=0, k \neq n}^{N} \frac{D-k}{n-k}, \quad n=0,1, \cdots, N .
$$

In Fig. 3, the frequency-domain error and the group delay are compared for the sinc and Lagrange interpolation FD filters of order $N=11$ and their truncated versions of order $\nu=3,6,9$, where the desired delay $D=5.4$, and the frequency is normalized by the sampling rate $f_{s}$, i.e., the normalized frequency 0.5 pertains to the Nyquist limit. From this figure, we may find that as the truncated FD filter order $\nu$ decreases, the frequency-domain error increases, and the group delay deviates from the desired delay gradually.

As is shown in both paradigms, the head distortion is a serious problem specifically for the FD filters of a high order 


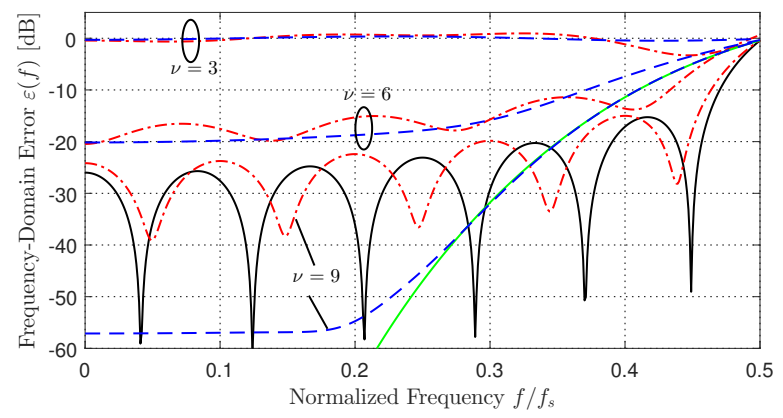

(a) Frequency-domain error $\varepsilon(f)$

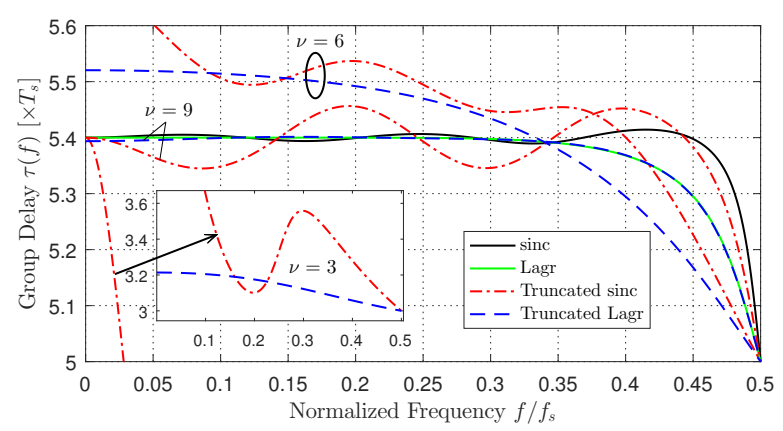

(b) Group delay $\tau(f)$

Fig. 3. Performance comparisons between classical sinc/Lagrange interpolation FD filters of order $N=11$ and their truncated versions of order $\nu=3,6,9$, for the desired delay $D=5.4$.

and the input sequence of short length.

Mathematically, the head distortion can be bypassed if the first $N$ (or at least $\lceil N / 2\rceil$ ) output samples are removed, i.e., by simply setting them to zero. However, in practice, these samples cannot be removed, because they carry important information and the removal is also a type of distortion. As shown in Fig. 2, if the desired output $\tilde{y}(l)$ is obtained by a CCFD transceiver, the SI caused by the input $x(l)$ will be effectively cancelled. If the head part of the output $y_{\text {sinc }}(l)$ is removed, the SI cancellation capability will be very likely worse than that without the removal.

\section{DisTORTION REDUCTION}

To reduce the head distortion of FD filters, we propose an interpolation scheme by varying the filter's impulse response with the output sample index. The first $N$ output samples of the varied-response FD filter can be written as

$$
y(l)=\sum_{n=0}^{N} x(N-n) h_{\mathrm{V}}(n, \hat{l}+D), \quad l=0,1, \cdots, N-1,
$$

where $x(N-n)$ is an input sample, and $h_{\mathrm{V}}(n, \hat{l}+D)$ with $\hat{l}=N-l$ is the $n^{\text {th }}$ coefficient of the varied-response FD filter for the $l^{\text {th }}$ output sample with a desired delay $D$.

The proposed FD filter design for a desired delay $D$ is expressed as

$$
\hat{h}(n, \hat{l}+D)= \begin{cases}h_{\mathrm{V}}(n, \hat{l}+D), & 0 \leqslant l \leqslant N-1, \\ h(n, D), & N \leqslant l \leqslant L,\end{cases}
$$

where $\hat{h}(n, \hat{l}+D)$ denotes the $n^{\text {th }}$ coefficient of the proposed FD filter for the $l^{\text {th }}$ output sample with a desired delay $D$,

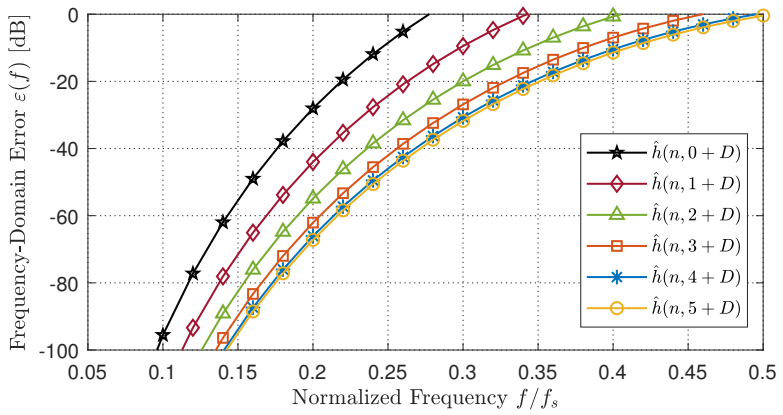

(a) Frequency-domain error $\varepsilon(f)$

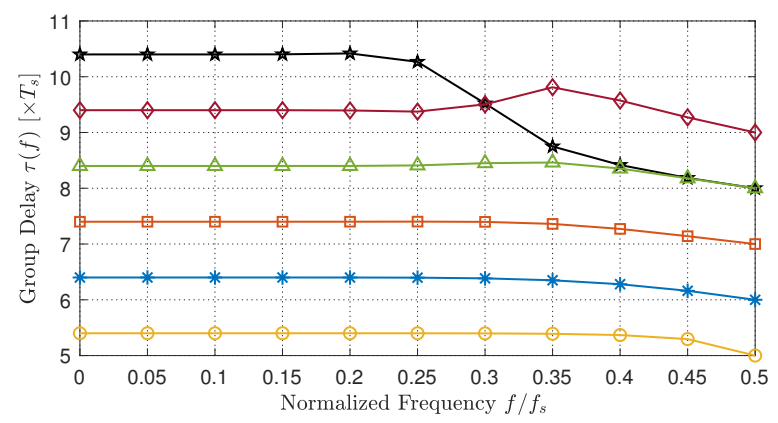

(b) Group delay $\tau(f)$

Fig. 4. Performance of the proposed FD filter with coefficients $h_{\mathrm{V}}(n, \hat{l}+$ $D)=h_{\text {Lag }}(n, \hat{l}+D)$ for the filter's order $N=11$ and the desired delay $D=5.4$.

and $h(n, D)$ denotes the $n^{\text {th }}$ coefficient of the original nontruncated FD filter for the desired delay $D=\lfloor D\rfloor+\alpha$ with an arbitrary interpolation method, $n=0,1, \cdots, N$. Note that, the varied-response FD filter's coefficients $h_{\mathrm{V}}(n, \hat{l}+D)$, $n=0,1, \cdots, N$, can be formed with an arbitrary interpolation method as well. Moreover, $h_{\mathrm{V}}(n, \hat{l}+D)$ and $h(n, D)$ are unnecessarily formed with the same interpolation method.

Then, the output of the proposed FD filter can be written in a vector form as

$$
\hat{\mathbf{y}}=\operatorname{diag}(\hat{\mathbf{X}} \hat{\mathbf{H}}),
$$

where the function $\operatorname{diag}(\cdot)$ returns a column vector of the main diagonal elements in a square matrix, and the $(L+1) \times 1$ column vector $\hat{\mathbf{y}}=[\hat{y}(0), \hat{y}(1), \cdots, \hat{y}(L)]^{\mathrm{T}}$ contains the improved output samples. The $(L+1) \times(N+1)$ matrix $\hat{\mathbf{X}}$ contains the input samples and its $l^{\text {th }}$ row is given by

$$
\hat{\mathbf{x}}_{l .}=\left\{\begin{array}{lr}
{[x(N), x(N-1), \cdots, x(0)],} & 0 \leqslant l \leqslant N-1, \\
{[x(l), x(l-1), \cdots, x(l-N)],} & N \leqslant l \leqslant L .
\end{array}\right.
$$

The $(N+1) \times(L+1)$ matrix $\hat{\mathbf{H}}$ contains the FD filter coefficients given in (9), and its $l^{\text {th }}$ column is

$$
\hat{\mathbf{h}}_{\cdot l}=[\hat{h}(0, \hat{l}+D), \hat{h}(1, \hat{l}+D), \cdots, \hat{h}(N, \hat{l}+D)]^{\mathrm{T}} .
$$

As the calculation of our FD filter coefficients is varied with the input sample index $l$, we compare the frequency-domain error and the group delay of the proposed filter $\hat{h}(n, \hat{l}+D)$ in Fig. 4 for $\hat{l}=0,1, \cdots, 5$, i.e., $l=11,10, \cdots, 6$, where the filter's order $N=11$ and the desired delay $D=5.4$. Both the varied-response FD filter and the original nontruncated FD filter are formed by the Lagrange interpolation, 
i.e., $h_{\mathrm{V}}(n, \hat{l}+D)=h_{\mathrm{Lag}}(n, \hat{l}+D)$ and $h(n, D)=h_{\mathrm{Lag}}(n, D)$, with $h_{\mathrm{Lag}}(n, \cdot)$ given in (7). In contrast to the performance of truncated FD filters in Fig. 3, we may find that the frequencydomain error is dramatically reduced by our solution, especially in the low-frequency region. In addition, Fig. 4(b) implies that the minimization of head distortion is equivalent to the varied-response FD filter design for the desired delay $\hat{l}+D, \hat{l}=1,2, \cdots, N$.

\section{Performance EVAluation}

As discussed above, the head distortion increases as the FD filter order gets higher. In this section, we evaluate the performance gain obtained by our solution over traditional FD filters for a practical scenario of CCFD communications, where high-order FD filters are needed for the SI cancellation.

The time-domain samples of an SI signal at the receiver port of a CCFD transceiver are expressed as

$$
y_{\mathrm{SI}}(l)=A s(l-\tau), \quad l=0,1, \ldots, L,
$$

where $A$ and $\tau$ are the attenuation and the delay, respectively, in the channel from the transmitter port to the receiver port of the CCFD transceiver. The transmitted signal $s(t)$ is bandlimited in the range of $\left[0, f_{0}\right]$ and sampled at the rate $f_{s}$ to form the samples $s(l)$, with the oversampling ratio $R=f_{s} /\left(2 f_{0}\right)$.

To cancel the SI, the transceiver reconstructs a delayed version of the transmitted signal at its receiver port as $y_{\mathrm{RE}}(l)=$ $\hat{A} \hat{s}(l)$, where $\hat{A}$ is the estimated SI channel attenuation, and the FD filter output $\hat{s}(l)=s(l-\hat{\tau})$ with the delay $\hat{\tau}=\lfloor\hat{\tau}\rfloor+\alpha$. Assuming the SI channel state information is perfectly known by the transceiver, we have $\hat{A}=A$ and $\hat{\tau}=\tau$.

For quantifying the performance of different FD filters in the reconstruction of delayed signals, the relative RMS error of a reconstructed signal in comparison to the received signal is defined as

$$
\varepsilon_{\mathrm{r}}=\frac{\left\|\mathbf{y}_{\mathrm{RE}}-\mathbf{y}_{\mathrm{SI}}\right\|}{\left\|\mathbf{y}_{\mathrm{SI}}\right\|}
$$

where $\mathbf{y}_{\mathrm{RE}}$ and $\mathbf{y}_{\mathrm{SI}}$ are the vector forms of $y_{\mathrm{C}}(l)$ and $y_{\mathrm{SI}}(l)$, respectively, $l=\lfloor D\rfloor-1,\lfloor D\rfloor, \cdots, L$. Moreover, the relative RMS error achieved by traditional FD filters given in (3) is denoted by $\varepsilon_{\mathrm{r}}^{\mathrm{T}}$ and that achieve by our proposed solution given in (10) is denoted by $\varepsilon_{\mathrm{r}}^{\mathrm{P}}$. For a thorough inspection, the relative RMS error in the case of ideal FD filters without truncation, denoted by $\varepsilon_{\mathrm{r}}^{\mathrm{NT}}$, is investigated as well.

Next, three types of traditional FD filters, i.e., sinc, Farrow [19] and Lagrange, are applied in the SI signal reconstruction with a desired FD $\alpha=0.5$. Our proposed solution with the coefficient $h_{\mathrm{V}}(n, \hat{l}+D)=h_{\mathrm{Lag}}(n, \hat{l}+D)$ is used to reduce the head distortion.

In Fig. 5, the relative RMS error versus the FD filter's order $N$ is investigated, where the oversampling ratio $R=4$ and the input length $L=70 R$, i.e, composed of 70 symbols. As shown in this figure, the performance of our solution approaches that of the ideal case without truncation, i.e., $\varepsilon_{\mathrm{r}}^{\mathrm{P}} \approx \varepsilon_{\mathrm{r}}^{\mathrm{NT}}$. Besides, both the performance gain of our solution, i.e., $\varepsilon_{\mathrm{r}}^{\mathrm{T}}-\varepsilon_{\mathrm{r}}^{\mathrm{P}}$, and the impact of head distortion, i.e., $\varepsilon_{\mathrm{r}}^{\mathrm{T}}-\varepsilon_{\mathrm{r}}^{\mathrm{NT}}$, are improved with the increase in $N$. More specifically, the performance gains achieved by our solution are $0.2 \mathrm{~dB}, 6.2 \mathrm{~dB}$ and $7.3 \mathrm{~dB}$ over traditional sinc, Farrow and Lagrange FD filters.

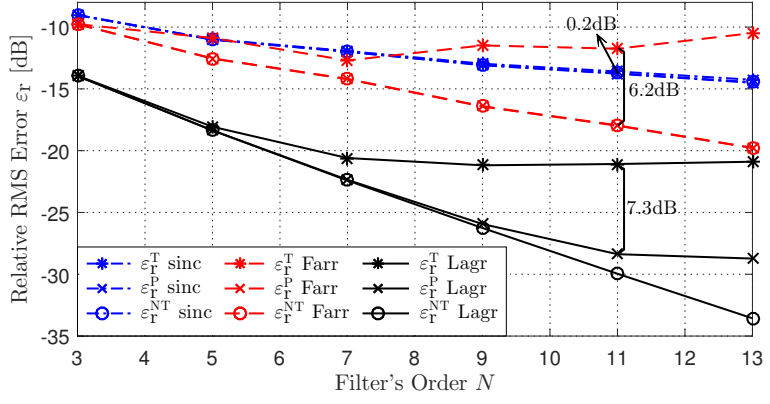

Fig. 5. Relative RMS error comparisons between our solution and traditional FD filters, including sinc, Farrow and Lagrange, for the desired FD $\alpha=0.5$, with the oversampling ratio $R=4$ and the input length $L=70 R$.

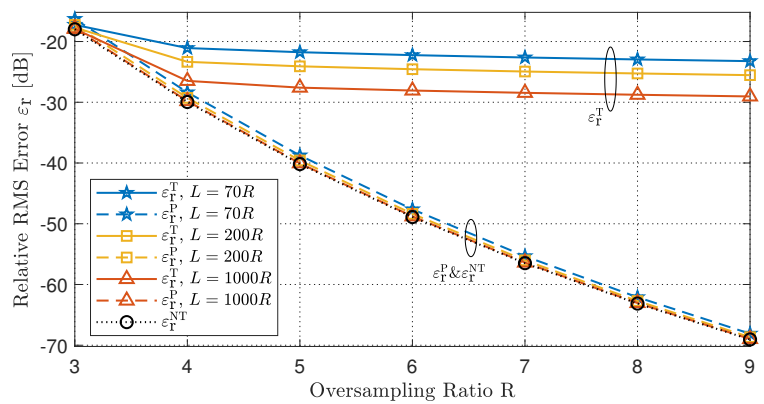

Fig. 6. Relative RMS error achieved by Lagrange FD filters of order $N=11$ versus the oversampling ratio $R$, for the desired FD $\alpha=0.5$.

Furthermore, the impact of the oversampling ratio $R$ on the relative RMS error achieved by Lagrange FD filters of order $N=11$ is investigated in Fig. 6, where the desired FD $\alpha=0.5$. As expected, the shorter the input sequence is, the more the head distortion impacts on the overall output. This figure also reveals that the reduction of oversampling ratio will further improve the performance of our solution and decrease the head distortion in traditional FD filters.

\section{CONCLUSION AND Discussion}

In this paper, the problem of head distortion and its causes were identified in FD filters, inspired by which a novel FD filter design was proposed to solve this problem and eventually reduce the overall distortion. Illustrative numerical results substantiated the validity and feasibility of the proposed design, specifically when the input sequence is short.

The proposed scheme has two limits, though it solves the head distortion problem efficiently. Firstly, its performance gain in the high-frequency region is lower than that in the low-frequency region. Secondly, its computational complexity is a bit higher than that of conventional FD filters. In detail, our solution needs $N^{2}$ multiplications and $N^{2}-N$ additions to deal with the distortion in the first $N$ output samples, whilst the convolution operation in conventional design needs $\left(N^{2}+N\right) / 2$ multiplications and $\left(N^{2}-N\right) / 2$ additions. Hence, better performance gain in high-frequency bands and further lower computational complexity are to be pursued in our future works. Moreover, the generalisation of our solution to any type of FIR filters, i.e., not limited to FD filters, will also be investigated in our future work. 


\section{REFERENCES}

[1] T. I. Laakso, V. Valimaki, M. Karjalainen, et al, "Splitting the unit delay [FIR/all-pass filters design]," IEEE Signal Process. Mag., vol. 13, no. 1, pp. 30-60, Jan. 1996.

[2] A. Zeineddine, A. Nafkha, S. Paquelet, et al, "Comprehensive Survey of FIR-based Sample Rate Conversion,” J. Signal Process. Syst., vol. 92, no. 7, pp. 1-13, July. 2020.

[3] S. Srivastava, A. K. Dwivedi, and D. Nagaria, "Optimal design of symmetric fractional delay filter using firefly algorithm," Int. J. Circ. Theor. Appl., vol. 48, pp. 1511-1522, Aug. 2020.

[4] V. Valimaki and T. I. Laakso, "Principles of fractional delay filters," in Proc. IEEE Int. Conf. Acoustics, Speech, and Signal Process., Istanbul, Turkey, Jun. 2000, vol.6, pp. 3870-3873.

[5] D. Luengo, C. J. Pantaleon, J. Ibanez, et al, "Design of simultaneous sampling systems based on fractional delay Lagrange filters," IEEE Trans. Circuits Syst. II, Analog Digit. Signal Process., vol. 47, no. 5, pp. 482-485, May 2000.

[6] V. Valimaki and A. Haghparast, "Fractional Delay Filter Design Based on Truncated Lagrange Interpolation," IEEE Signal Process. Lett., vol. 14, no. 11, pp. 816-819, Nov. 2007.

[7] J. T. Olkkonen and H. Olkkonen, "Fractional Time-Shift B-Spline Filter," IEEE Signal Process. Lett., vol. 14, no. 10, pp. 688-691, Oct. 2007.

[8] J. T. Olkkonen and H. Olkkonen, "Fractional Delay Filter Based on the B-Spline Transform," IEEE Signal Process. Lett., vol. 14, no. 2, pp. 97-100, Feb. 2007.

[9] C. W. Farrow, "A continuously variable digital delay element," in Proc. IEEE Int. Symp. Circuits Syst., Jun. 1988, pp. 2641-2645.

[10] S. Paquelet, A. Zeineddine, A. Nafkha, et al, "Convergence of the Newton Structure Transfer Function to the Ideal Fractional Delay Filter," IEEE Signal Process. Lett., vol. 26, no. 9, pp. 1354-1358, Sept. 2019.

[11] S. Paquelet, A. Zeineddine, A. Nafkha, et al, "Convergence of the Newton Structure Transfer Function to the Ideal Fractional Delay Filter," in Proc. 25th Int. Conf. Telecom., St. Malo, 2018, pp. 98-103.

[12] G. Braileanu, "Extended-window interpolation applied to digital filter design," IEEE Trans. on Signal Process., vol. 44, no. 3, pp. 457-472, Mar. 1996.

[13] C. Li, H. Zhao, F. Wu and Y. Tang, "Digital Self-Interference Cancellation With Variable Fractional Delay FIR Filter for Full-Duplex Radios," IEEE Commun. Lett., vol. 22, no. 5, pp. 1082-1085, May 2018.

[14] M. Duarte, C. Dick and A. Sabharwal, "Experiment-Driven Characterization of Full-Duplex Wireless Systems," IEEE Trans. on Wireless Commun., vol. 11, no. 12, pp. 4296-4307, Dec. 2012.

[15] M. Ma, S. Tian, Y. Chen, L. Wang, Y. Yang, L. Wan; B. Jiao and H. V. Poor, "A Prototype of Co-Frequency Co-Time Full Duplex Networking," IEEE Wireless Commum. Mag., vol. 27, no. 1, pp. 132-139, Feb. 2020.

[16] S. Tian, M. Ma, Y. Yang and B. Jiao, "Blind Analog Interference Cancellation," IEEE Commun. Lett., vol. 21, no. 8, pp. 1867-1870, Aug. 2017.

[17] D. Yavuz, "Meteor burst communications," IEEE Commum. Mag., vol. 28 , no. 9 , pp. 40-48, Sept. 1990.

[18] N. Safari, J. P. Tanem and T. Roste, "Block based predistortion for amplifier linearization in burst type mobile satellite communications," in The European Conf. on Wireless Tech., Paris, 2005, pp. 325-328.

[19] A. Tarczynski, G. D. Cain, E. Hermanowicz and M. Rojewski, "WLS design of variable frequency response FIR filters," in Proc. IEEE Int. Symp. Circuits Syst., Hong Kong, Jun. 1997, vol. 4, pp. 2244-2247. 